\section{Wnt it rains}

\section{By Steve Edelson, Executive Editor}

For the second time in as many months, a research group has found a new target in the Wnt pathway that could be more druggable than previously identified components of the cancer-inducing signaling cascade. Antagonizing the target, a kinase called TNIK, might be particularly useful in treating colon cancer, but its general expression patterns will have to be analyzed to evaluate the potential side effects of inhibiting it.

In most colon cancers, the Wnt pathway malfunctions via aberrant expression of $\beta$ catenin-the pathway's key transcription factor. However, most interactions between Wnt signaling molecules are protein-protein and thus are hard to block with small molecules.

In September, a group at Novartis AG's Novartis Institutes for Biomedical Research identified two new enzymes in the pathway: tankyrase TRF1-interacting ankyrin-related ADP-ribose polymerase (TNKS) and TNKS2, which help degrade a complex of molecules that regulates $\beta$-catenin. ${ }^{1}$ The pharma company is developing small molecule inhibitors of these enzymes.

Now, Dutch researchers have added a downstream target-TRAF2 and NCK interacting kinase (TNIK) - to the mix. Using proteomics, the group showed that TNIK binds to the transcription factor TCF4, which, along with $\beta$-catenin, is one of the main molecular effectors of the Wnt

Figure 1. New druggable targets in the Wnt pathway. The recent identification of three new players in the Wnt pathway, tankyrase TRF1-interacting ankyrin-related ADP-ribose polymerase ( TNKS), TNKS2 and TRAF2 and NCK interacting kinase (TNIK), provides a trio of druggable targets in a signaling cascade that companies have shied away from because of the difficulty of disrupting proteinprotein interactions with small molecules.

Novartis AG is already developing small molecule inhibitors of TNKS and TNKS2 to target these two enzymes in the cytosol, where they help break up the multiprotein degradation complex (MDC), a group of molecules that regulates $\beta$-catenin.

Researchers believe that the best place to antagonize TNIK is likely to also be the cytosol, where the kinase is thought to bind to $\beta$-catenin and then translocate to the nucleus. Once in the nucleus, TNIK binds to another molecular effector of the Wnt signal - TCF4. No compounds are yet in development to target TNIK. signal in the small intestine ${ }^{2}$ (see Figure 1, "New druggable targets in the Wnt pathway").

TNIK could be attractive to drug developers for two reasons: it is a kinase and thus potentially amenable to inhibition by small molecules, and it is expressed in the intestinal crypt, an area of the intestinal epithelium in which colorectal tumors originate.

"We're always interested in enzymes to inhibit, so we took a mass [spectrometry] approach to find targets" in the Wnt pathway, said Tokameh Mahmoudi, lead author of a paper in The EMBO Journal describing the results. "This was a great hit. At first we were surprised because all the available information about this kinase shows that it's cytoplasmic and involved in cell adhesion."

Mahmoudi did the work as a postdoc in the laboratory of Hans Clevers, director of the Hubrecht Institute. She is now an assistant professor of biochemistry at Erasmus Medical Center.

Following the proteomics-based identification of TNIK, the next step was finding the functional relevance of the kinase. "It does have other functions in cell adhesion, but its transcriptional function is essential and seems to be exclusive to Wnt signaling, at least in the systems we've looked in," said Mahmoudi.

Cell line experiments showed that "TNIK is localized to the nuclei of Wnt-active intestinal crypts and is recruited to promoters of Wnt target genes in mouse crypts and in colorectal cells in a $\beta$-catenin-dependent manner," according to the authors of the EMBO Journal paper.

"Another nice thing is that TNIK is not so pleiotropic," added Mahmoudi. "Our array data showed that this is specific for Wnt target genes," unlike other enzymes that can be involved in hundreds of transcriptional pathways.

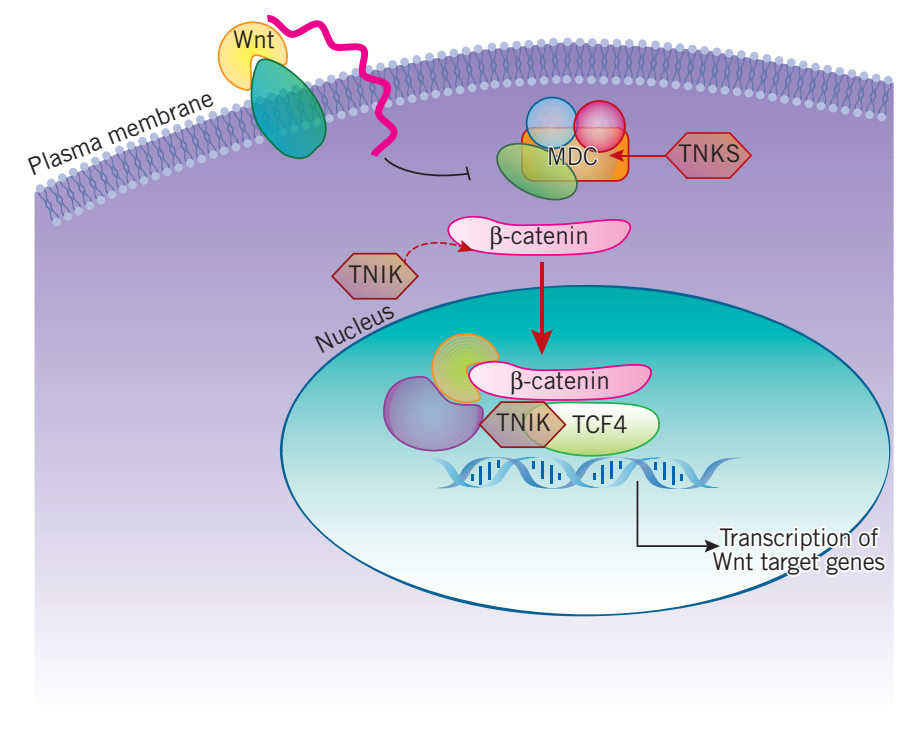




\section{TARGETS \& MECHANISMS}

Although TNIK exerts its effects on the Wnt pathway in the nucleus, an area that is hard for small molecules to access, the kinase could be targeted when it is in the cytosol, thereby preventing its translocation into the nucleus.

"If you interfere with this process, it's one step toward inhibiting Wnt target gene activation," noted Mahmoudi. "A next step could be designing a specific small molecule inhibitor for TNIK."

TNIK is "very amenable to a small molecule," said John Hood, CSO of Wintherix LLC. "This target hasn't gotten any attention yet. We will be taking at look at this kinase. It doesn't change our own lead programs but it doesn't hurt to have backups."

Wintherix has two chemical series in preclinical development for cancer. The compounds hit undisclosed targets in the Wnt pathway, and Hood said the company expects to nominate a lead candidate for INDenabling studies by January 2010.

\section{Tumor specificity}

Although TNIK represents a druggable target in the relatively intractable Wnt pathway, a key question is whether the kinase is active only in tumor tissues or more generally throughout the body. If the latter turns out to be the case, interfering with its activity could cause unwanted side effects.

"As far as we see, TNIK is enriched in Wnt-active tissues and most importantly it's nuclear in Wnt-active tissues," said Mahmoudi. "But we haven't yet looked at its general expression."

Those experiments will be important, said Randall Moon of the University of Washington, to help "determine whether this kinase is involved in Wnt signaling in a broad range of tissues or just in the contexts in which they study it, which is a limited number of cell lines."
Moon is a professor of pharmacology at University of Washington. $\mathrm{He}$ is focused on Wnt signal transduction and is a scientific cofounder of Fate Therapeutics Inc., a biotech focused on stem cell therapeutics.

In May, Moon's group at the university reported that Bruton's tyrosine kinase (BTK) is a negative regulator of Wnt signaling. ". "Some of the same cautionary concerns" about tissue selectivity apply there as well as with TNIK, he told SciBX.

Moon wanted to know whether TNIK's function is "really restricted to just modulating Wnt signaling or is it involved in other cellular processes which may not have been revealed by the assays conducted." $\mathrm{He}$ suggested looking at what happens when TNIK is systemically attenuated or blocked in mice or zebrafish.

Clevers told SciBX the Hubrecht researchers have submitted a patent application covering TNIK to block the Wnt cascade in colon cancer and other indications.

Edelson, S. SciBX 2(42); doi:10.1038/scibx.2009.1555

Published online Oct. 29, 2009

\section{REFERENCES}

1. Huang, S. et al. Nature 461, 614-620 (2009)

2. Mahmoudi, T. et al. EMBO J.; published online Oct. 15, 2009; doi:10.1038/emboj.2009.285

Contact: Hans Clevers, Hubrecht Institute, Utrecht, the Netherlands e-mail: h.clevers@niob.knaw.nl

3. James, R.G. et al. Sci. Signal. 2, ra25 (2009)

\section{COMPANIES AND INSTITUTIONS MENTIONED}

Erasmus Medical Center, Rotterdam, the Netherlands Fate Therapeutics Inc., La Jolla, Calif.

Hubrecht Institute, Utrecht, the Netherlands

Novartis AG (NYSE:NVS; SIX:NOVN), Basel, Switzerland

University of Washington, Seattle, Wash.

Wintherix LLC, San Diego, Calif. 